\title{
A relationship between personality and empathy in teenagers' school sports club participation
}

\author{
Se Jeong Kwon* \\ College of Sports Science, Chung-Ang University, Anseong, Korea
}

This study aims to identify the relation between students' participation in sports club activities and personality and sympathy based on the findings obtained from personalty and sympathy study and understand what the interrelational characteristics are. This study investigated a total of 408 individuals (male, 58.3\%; female, $41.7 \%$ ) who have continuously participated in sports club activity in middle schools located at Seoul and Gyeonggi province, Korea. To look at the type of event that they selected, 127 individuals (31.1\%) reported that "School sports club event is already defined in school." and 281 (68.9\%) reported that "We can select among several school sports club events." The events that they participated in are that 79 individuals participated in basket ball, 47 in line dancing, 96 in badminton, 2 in yoga, 31 in football, 129 in pingpong, and 20 in floorball. In conclusion adolescence is a period that ad- olescents are physically and mentally developed in terms of personality, morality, creativity, and sociality. During this period, learning and experience are very important for lifetime. Therefore, teenagers' school sports club activity can contribute to fostering positive and desirable personality and stabilizing emotional empathy among student participants. This study identified the relation between personality and empathy in adolescents, which was not studied before. As presented in previous researches, it can be found that positive personality can be cultivated by teenagers' communal activities in school life. The link to connect this with empathy factor is considered to be the very communal activity, sports.

Keywords: Personality, Empathy, Teenager, School sports club

\section{INTRODUCTION}

Korean teenagers' school violence and game addiction are emerging as serious social problems and subsequently, the suicide rate caused by friendly relations and intense academic stress is gradually increasing. The countermeasure taken by the government to solve such problems among teenagers is to improve students' personality. Leming (2000) proposed that for an effective personality education, it should be conducted consistently across the entire areas of curriculum. Therefore, determining that personality education using hours of participating in physical activities that they find most interesting is very effective for Korean teenagers, they included information on personality education program utilizing physical activity in the entire secondary school curriculum (Ministry of Education, 2013). In other words, they included school sports club activities into formal secondary school education under the purpose that physical activity is important to prevent suicide caused by game addiction, school violence, bullying, and academic stress among secondary school students.

As a matter of fact, personality education has been performed in Korea, but only focused on giving basic etiquette lessons. Due to lack of teenagers' social skills, community spirit, and consideration of others, the necessity of reviewing its effectiveness has been raised (Ministry of Education, 2010). In other words, deliberate educational approach is required to foster good personality (Bierhoff and Rohmann, 2004). Therefore, it's considered that personality education using physical activities in which teenagers are highly interested in (Wankel and Kreisel, 1985) will be effective and that school sports clubs in which students will participate voluntarily will be more effective. This is why it's considered that attempts to perform deliberate personality education through school sports club will increase the effectiveness.
*Corresponding author: Se Jeong Kwon (ib https://orcid.org/0000-0003-0795-9962 College of Sports Science, Chung-Ang University, 4726 Seodong-daero, Daedeok-myeon, Anseong 17546, Korea

Tel: +82-31-670-3281, Fax: +82-31-675-1326, E-mail: foreverksj@cau.ac.kr Received: June 26, 2018 / Accepted: August 13, 2018
This is an Open Access article distributed under the terms of the Creative Commons Attribution Non-Commercial License (http://creativecommons.org/licenses/by-nc/4.0/) which permits unrestricted non-commercial use, distribution, and reproduction in any medium, provided the original work is properly cited. 
In Korea, they have not put stress on physical education (PE) class due to entrance examination-oriented educational climate. However, they are trying to increase students' participation in school sports clubs (Ministry of Education, 2013) including placement of PE-specialized teachers into schools and support of sports club league along with school PE activation policy. Accordingly, they spare no efforts to support the activation of school sports club in Metropolitan and Provincial Offices of Education across the country and provide students with effective and concrete personality education plans by distributing the personality-enhancing teaching materials including school sports club activity to local school fields. With respect to the effectiveness of school sports club, students who participated actively in such clubs were found to develop positive personality and sociality and build an upright character in addition to physical strength enhancement and good health maintenance.

In fact, to develop good personality, comprehensive and deliberate educational approach is required by families and societies (Bierhoff and Rohmann, 2004; Lickona, 1996). So far, personality education has been conducted in Korea, but students who received such an education lacked sociality, community spirit, and consideration of others due to simply basic etiquette-oriented personality education policy. It raised the necessity of reviewing the effectiveness (Ministry of Education, 2010). This is why it seems that deliberate efforts to perform personality education through school sports club activity will increase the effectiveness.

However, teenagers' all problems cannot be solved by the current personality education-centered practice. In other words, the use of various personality-related variables will enable us to verify the effect of such a personality education. Among which, the importance of personality-based empathic ability has recently been emerging. In other words, one of the virtues that are valued with upright character to teenagers is the very sympathy, an ability to form a positive relation with others (Barr and Higgins-D'Alessandro, 2007). In other words, if an individual's adaptive behavior in community life is personality, the empathic ability to feel and accept other people's feelings (Preston and de Waal, 2002) is an essential factor to form a positive interpersonal relation.

In Canada, Gordon (2009) conducted a program called as 'roots of sympathy' through which kindergartens and elementary, middle, and high school students watched babies in local region growing. After participating in this program, students could develop an ability to understand others and take care of the under-privileged. Furthermore, it's also reported in a study on elementary school students that the group of students who received brain education-related personality education lessons showed a higher empathic ability than the group who did not. Thus, it's expected that the characteristics of sympathy which is being studied as a concept to accept perspective on others and include sympathetic feeling, emotional contagion, and helping behavior can be improved by participating in sports club activities and fostering the personality of participants.

In other words, as sports activity participation can expect physical, psychological, and social effects, personality and empathic ability improvement can also be expected (Gano-Overway et al., 2009), for this is effective for understanding and being considerate of others and maintaining and enhancing interpersonal relations in the process of being involved in sports activities. Therefore, teenagers' sports participation can help to foster appropriate personality and empathic ability as member of future society and reduce social problems including school violence and bullying (Gini et al., 2007).

Therefore, this study aims to identify the relation between students' participation in sports club activities and personality and sympathy based on the findings obtained from personalty and sympathy study and understand what the inter-relational characteristics are. In particular, this study measured the level of involvement to identify the degree of participants' participation in school sports club after school unlike the previous researches. Many previous researches on the degree of participation in school sports club regarded the degree, frequency, and period of participation as intensity of participation. However, in this study, the level of involvement that participants recognize by themselves directly is considered to measure the degree of participation more exactly than the degree of participation that students simply participate in after-school sports club classes with an well-organized curriculum compared to other physical activities. Involvement is a concept to measure the importance that an individual feels about a situation in a particular situation and the degree of interest. Therefore, this study defined the degree of participation as level of involvement after going through a meeting of experts consisted of major professors (sociology, pedagogy) and major doctors.

The previous researches reported that there was a difference in the level of sympathy depending on the degree of participation. In other words, there will be a difference in relational characteristics between personality and sympathy depending on the level of sports involvement. However, there are limitations in understanding the characteristics of personality and sympathy due to lack of previous researches that may support such a prediction. Therefore, this study aimed to identify what relation personality and sympa- 
thy may have depending on middle school students' sports club participation as clearly as possible. To analyze the relation between personality and sympathy depending on the involvement of school sports club participants based on the above purposes and research problems, this study set up the following research hypotheses.

1-1. Confidence may have an impact on subfactors of sympathy such as positive sharing, emotional contagion, and emotional involvement depending on the level of school sports club participants' involvement.

1-2. Positive attitude may have an impact on subfactors of sympathy such as positive sharing, emotional contagion, and emotional involvement depending on the level of school sports club participants' involvement.

1-3. Community spirit may have an impact on subfactors of sympathy such as positive sharing, emotional contagion, and emotional involvement depending on the level of school sports club participants' involvement.

\section{Participation in school sports club}

In Korea, after-school PE activities were limited. This is because Korea did not put stress on PE class due to entrance examination-oriented educational climate. However, as school PE activation policy, many efforts to increase students' participation in school sports club including placement of PE-specialized teachers and support of school sports club leagues (Ministry of Education, 2013) have been made. School sports club was actively enforced to solve school problems and obesity issues, foster their sociality, and promote school athletic clubs (Ministry of Education, 2008). Therefore, all students in elementary, middle, and high schools came to participate in school sports club as they wanted, and in particular school sports club activities have been included into middle schools' formal education course since the 2nd semester of 2012 and operated characteristically to the educational purposes.

Currently, the number of students who participated in after-school sports club increased from $17.1 \%$ in $2008,27.4 \%$ in $2009,37.6 \%$ in 2010, $45.0 \%$ in 2011, to $84.2 \%$ in 2012 (Ministry of Culture, Sports and Tourism, 2013). The school sports club events were registered with 103 events in total to the school sports club system as of December, 2012 and the number of clubs reached 172,002 clubs $(90,620$ in elementary school, 51,951 in middle school, and 29,388 in high school). In addition, to look at the current status of students' participation in national school sports club competitions in 2012, a total of 15,035-365 teams from elementary school, 421 teams from middle school, and 239 teams from high school—participated. This suggests that as part of school PE activation policy, students' participation in school sports club is increasing rapidly.

\section{Sports participation and personality}

Personality includes character, nature, propensity, behavior, and attitude and is a psychological system that determines an individual's thought, emotion, and behavior. Personality depends on behaviors in the process of life or circumstances (Caspi and Roberts, 2001). It is also similar to the concept of sociality in the form of an individual's adaptive behavior in the space of communal living (Roberts et al., 2003).

To look at the researches on the relationship between sports participation and personality, sport participation was reported to be effective for fostering teenagers' personality. In other words, participation in school sports club in middle school is an opportunity to grow into a person who has ultimately right personality developing sound character and fostering good sociality through group activity in addition to enhancement of physical strength and maintenance of good health by working out hard among students. Also, in Korea, model PE manual for practicing personality has been developed and distributed across the nation to foster right personality among students, and thus appropriate tools to evaluate this were required. Newcombe and Boyle (1995) proposed that personality appeared differently depending on sports participation or nonparticipation and there occurred a difference between type of sports and gender. Like this, researches on personality cultivation through sports participation have been continuously conducted in sociopsychological area of sports and interests in humanity recovery through sports participation become hight and thus the necessity of personality education is also increasing (Anderson, 2001). For this, the direction of personality education should be toward altruistic personality and cultivating the personality that one can be sympathetic with the pain of others is important, and according to the norms presented by family and school, both family and community need to take interest in personality education since childhood.

\section{Empathy skills}

Empathy is a concept that accepts perspective of others and includes sympathy, emotional contagion, and helping behavior and thus a mood or feeling that one can feel the same as the other people in terms of opinion, emotion, thought, or argument (Donnerstein et al., 1975). Empathy is also a social ability of an individual (Decety and Jackson, 2004). It has a motivational influence on 
human interactions, which adds quality in every workplace (Gini et al., 2007). Like this, empathy is an essential factor to live together with others and thus regarded as important factor for improving communicative ability in modern society. Empathy suppresses threatening behavior, induces altruistic behavior, and plays a deciding role in fostering emotional bond among group members (Singer, 2006). Therefore, Gordon (2009) saw sympathy as foundation for personality education as empathy skills allow for having a mind to respect others' feelings and be considerate of others.

To look at the previous studies on empathy, empathy is defined as sharing of emotion, vicarious emotion, and emotional response that one corresponds with others' situation rather than one's own situation (Feshbach and Feshbach, 1982; Mehrabian and Epstein, 1972). Also, empathy related to interpersonal relation is significantly associated with interpersonal ability, altruistic behavior, sociality, and prosocial behavior like cooperativity (Eisenberg and Miller, 1987) and empathy skills in childhood are associated with social behavior (Findlay et al., 2006). Barr and Higgins-D'Alessandro (2007) reported that empathy and prosocial behavior are related and thus teenagers' empathy and prosocial factors are important factors to education for fostering positive school culture. Therefore, in order to solve such a problem as increased suicide rate caused by school violence and bullying which are increasing explosively in Korea, empathy is an essential factor. On the one hand, Gano-Overway et al. (2009) reported that sports program participation can improve empathy skills and adjust social behavior. Therefore, it can be said that empathy skills are changed by education and education through sports can improve empathy skills. In other words, seen from such previous researches, it can be predicted that sports activity participation during adolescent period can bring about positive empathy cultivation and improvement and personality is mutually related with empathy.

\section{MATERIALS AND METHODS}

\section{Participants}

This study investigated a total of 408 individuals (male, 58.3\%; female, $41.7 \%$ ) who have continuously participated in sports club activity in middle schools located at Seoul and Gyeonggi province, Korea. Among them, 213 individuals (52.2\%) were the 1st and 2nd graders and 195 (47.8\%) the 3rd graders. To look at the type of event that they selected, 127 individuals (31.1\%) reported that "School sports club event is already defined in school" and $281(68.9 \%)$ reported that "We can select among several school sports club events." The events that they participated in are that 79 individuals participated in basket-ball, 47 in line dancing, 96 in badminton, 2 in yoga, 31 in football, 129 in pingpong, and 20 in floorball.

\section{Mesurement of personality}

The measure of personality was used as a measure to represent the participation effect of school sports club the most among various personality factors and based on Cha et al. (2013) and Park and Heo (2012)'s personality scale for adolescents. This study selected the items that factorial wit was more than 0.5 in an exploratory factor analysis of personality scale and the cumulative distribution variance appeared at $63.54 \%$. In addition, Cronbach a test result of personality showed that confidence appeared at 0.83 , community spirit at 0.68 , and positive attitude at 0.78 . After then, confirmatory factor analysis (CFA) of personality scale was conducted finally to evaluate goodness of fit. As a result, the level was satisfactory $\left(\chi^{2} / d f=2.69\right.$, Tucker-Lewis index [TLI] $=0.924$, comparative fit index $[\mathrm{CFI}]=0.96$, root mean square error of approximation $[$ RMSEA $]=0.06$ ). This questionnaire consisted of 5-point scale (1, not at all; 5 , extremely so).

\section{Mesurement of empathy}

Empathy scale consists of 16 questions in total including three major factors - emotional and positive sharing, emotional contagion, and emotional involvement. It's based on Mehrabian and Epstein (1972)'s emotional empathy skills scale which were modified and complemented. As a result of Cronbach $\alpha$-test, reliability could be obtained as positive sharing appeared at 0.91 , emotional contagion at 0.84 , and emotional involvement at 0.80 and the cumulative distribution variance was $69.72 \%$. Convergent validity analysis and CFA of empathy scale were conducted to check the optimal status of construct concept and variable construction. After goodness of fit test, the level of goodness of fit for empathy scale was satisfied $\left(\chi^{2} / d f=2.403, \mathrm{TLI}=0.924, \mathrm{CFI}=0.935\right.$, RMSEA $=0.059)$. Also, convergent validity was found to be present because it satisfied the standard values of all evaluations (loading = 0.5 or higher, concept reliability $=0.7$ or higher). This questionnaire consisted of 5-point scale (1, not at all; 5, extremely so).

\section{Measurement of involvement}

To measure the involvement in school sports club, the questionnaire used in Wiley et al. (2000) was modified and supplemented for this study and the final questionnaire is composed of five questions. In this study, involvement is a concept that one can measure 
the importance that one feels at a particular situation and the degree of interest and thus refers to the level of involvement that one recognizes subjectively rater than simply determining whether one participated or not (Bennett et al., 2009). Therefore, measuring the intensity of involvement is effective for measuring the degree of sports participation as it represents every student's motivation for school sports club and association. As a result of Cronbach $\alpha$-test for participation scale, it appeared at 0.88 . As the level of school sports club involvement represents interest in and importance of sports club that student participants recognize, such a level will be effective for measuring the degree of participation. Therefore, the average value of participation factors (mean, 3.84) were obtained and then classified into three groups such as high involvement, intermediate involvement, and low involvement through frequency analysis (Wiley et al., 2000). This questionnaire consisted of 5-point scale (1, not at all; 5, extremely so).

\section{Data analysis and procedure}

This study manufactured a preliminary questionnaire based on previous researches and data and then produced a final questionnaire after testing validity and reliability. For questionnaire, this study selected after-school sports club participants from middle schools located at Seoul and Gyeonggi province as parent population and then selected research subjects by using a convenient sampling method. First, this study selected schools and then the researcher called PE teachers in middle schools to ask for cooperation and visited them with assistant researcher at a promised date of visit. They distributed the questionnaire to the students who participated in school sports club and then instructed them to complete it with self-administration method. The period of investigation was April to May in 2014 and prior to conducting a survey, students were sufficiently instructed about considerations when answering and intention and purpose of research. The time consumed for survey was about 15-20 min. and the questionnaires that students completed were collected immediately after the given time ended. A total of 408 questionnaires were finally selected except the questionnaires that were considered to be less reliable or regarded as unfaithful among the data collected. After the coding process of 408 questionnaires to a computer, questionnaire error or coding error was tested again and again. With this data, frequency analysis and descriptive analysis were conducted using IBM SPSS ver. 18.0 (IBM Co., Armonk, NY, USA). Also, to test the suitability of research hypothesis and research model, correlation analysis, CFA, and structural equation model (SEM) were conducted using AMOS 18.0 (Hannarea, Seoul, Korea). The

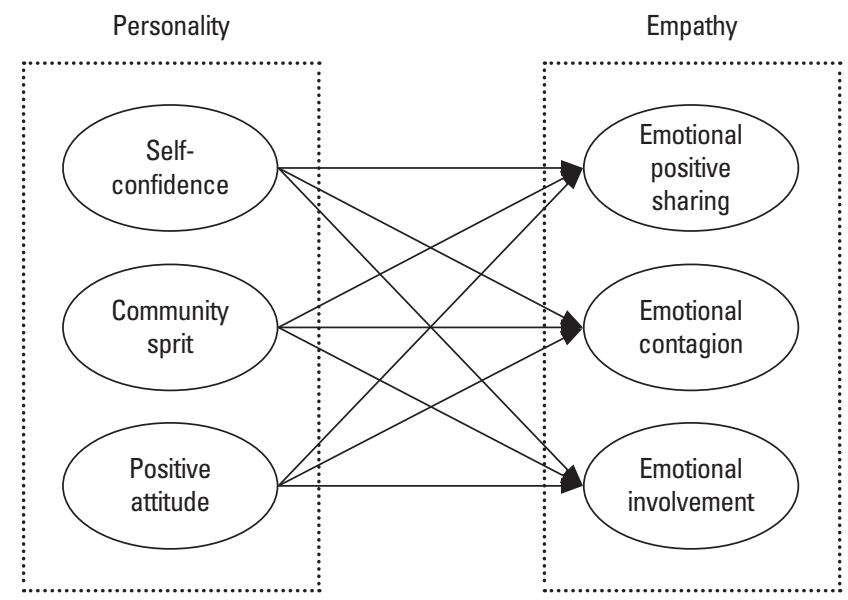

Fig. 1. Research model of personality factors and empathy factors in sports participation.

relational model of personality and empathy depending on sports participation is shown in Fig. 1.

\section{RESULTS}

\section{Descriptive statistics and correlation}

This study conducted correlation analysis in order to understand the degree of satisfying the discriminant validity between factors among the factors whose convergent validity was found and then correlation analysis. As shown in Table 1, as a result of correlation analysis, all relations between factors appeared in the same direction as the hypothesis-setting direction and mean and standard deviation of factors are presented. At this time, discriminant validity is present unless correlation coefficient between construct concepts is 1 at a confidence interval of $95 \%$. In addition, factors have discriminant validity if dismissing the null hypothesis that correlation is 1 (Anderson and Gerbing, 1988). Therefore, it can be seen that the scales used in this study have discriminant validity.

\section{Relations between personality and empathy}

This study conducted a structural model analysis to verify the goodness of fit model centering on the relation between personality and empathy. $\chi^{2}$ was at $533.254(d f=216, P=0.000)$ and thus appeared inappropriate as it was less than the standard value of $P$ $(P>0.05)$. However, the goodness of fit of $\chi^{2}$ was determined because it was pointed out that $\chi^{2}$ was sensitive to the size of sample and on the basis that goodness-of-fit indicators in SEM can be determined comprehensively with other indicators as they are relative indicators rather than absolute ones. Therefore, the goodness 
Kwon SJ • A relationship between personality and empathy

Table 1. Desciptive statistic and correlations for personality and empathy scale

\begin{tabular}{|c|c|c|c|c|c|c|}
\hline Variable & 1 & 2 & 3 & 4 & 5 & 6 \\
\hline 1. Self-condition & 1.000 & & & & & \\
\hline 2. Postive attitude & $0.534^{* *}$ & 1.000 & & & & \\
\hline 3. Community sprit & $0.603^{* *}$ & $0.456^{* *}$ & 1.000 & & & \\
\hline 4. Emotional positive sharing & $0.529^{* *}$ & $0.380^{* *}$ & $0.474^{* *}$ & 1.000 & & \\
\hline 5. Emotional contagion & $0.449^{* *}$ & $0.455^{* *}$ & $0.427^{* *}$ & $0.450^{* *}$ & 1.000 & \\
\hline 6. Emotional involvement & $0.496^{* *}$ & $0.501^{* *}$ & $0.490^{* *}$ & $0.598^{* *}$ & $0.666^{* *}$ & 1.000 \\
\hline Mean \pm SD & $3.79 \pm 0.69$ & $3.76 \pm 0.68$ & $4.05 \pm 0.66$ & $4.11 \pm 0.7$ & $3.65 \pm 0.84$ & $3.88 \pm 0.76$ \\
\hline
\end{tabular}

SD, standard deviation.

1-3: personality factors, 4-6: empathy factors.

${ }^{* *} P<0.01$.

Table 2. Hypothesis testing df the relation between personality and empathy

\begin{tabular}{lllcccc}
\hline Hypothesis & & & Path coefficient & Standard error & t-value & Adopted or dismissed \\
\hline Self-confidence & $\rightarrow$ & Emotional positive sharing & -0.257 & 0.209 & -1.230 & Dismissed \\
Self-confidence & $\rightarrow$ & Emotional contagion & -1.468 & 0.420 & $-3.493^{* * *}$ & Withheld \\
Self-confidence & $\rightarrow$ & Emotional involvement & -0.946 & 0.280 & $-3.374^{* * *}$ & Withheld \\
Positive attitude & $\rightarrow$ & Emotional positive sharing & 0.926 & 0.218 & $4.244^{* * *}$ & Adopted \\
Positive attitude & $\rightarrow$ & Emotional contagion & 2.626 & 0.465 & $5.646^{* * *}$ & Adopted \\
Positive attitude & $\rightarrow$ & Emotional involvement & 1.705 & 0.323 & $5.279^{* * *}$ & Adopted \\
Community spirit & $\rightarrow$ & Emotional positive sharing & 0.291 & 0.139 & $0.089^{*}$ & Adopted \\
Community spirit & $\rightarrow$ & Emotional contagion & -0.027 & 0.110 & Dismissed \\
Community spirit & $\rightarrow$ & Emotional involvement & 0.057 & 0.154 & 0.370 & Dismissed \\
\hline
\end{tabular}

${ }^{*} P<0.05$. ${ }^{* * *} P<0.001$.

of fit was verified through the numerical values of TLI, CFI, $\chi^{2} / d f$, and RMSEA that'd been adopted as criteria in CFA. As a result, $\mathrm{TLI}=0.921, \mathrm{CFI}=0.933, \chi^{2} / d f=2.445$, and RMSEA $=0.060$, which suggested that the goodness-of-fit was relatively well satisfied. Therefore, the research hypothesis and research model set in this study are relatively well explanatory of empirical data. To look at Table 2, hypothesis 1-1 'Confidence in school sports club participants will have an impact on positive sharing, subfactor of empathy' was dismissed because the path coefficient between two variables was at $-0.257(t=-1.230, P=0.219)$ and thus not statistically significant. The hypothesis 'Confidence in school sports club participants will have an impact on emotional contagion, subfactor of empathy' appeared statistically significant in a negative (-) way because the path coefficient between two variables was at $-1.468(t=-3.493, P<0.001)$ and thus withheld. Likewise, the hypothesis 'Confidence in school sports club participants will have an impact on emotional involvement, subfactor of empathy' appeared statistically significant in a negative (-) way because the path coefficient between two variables was at $-0.946(t=-3.374$, $P<0.001)$ and then withheld.

Hypothesis 2-1 'Positive attitude in school sports club partici- pants will have an impact on positive sharing, subfactor of empathy' appeared statistically significant because the path coefficient between two variables was at $0.926(t=4.244, P<0.001)$. 'Positive attitude in school sports club participants will have an impact on emotional contagion, subfactor of empathy' appeared statistically significant because the path coefficient between two variables was at $2.626(t=5.646, P<0.001)$ and so adopted. Also, 'Positive attitude in school sports club participants will have an impact on emotional involvement, subfactor of empathy' appeared statistically significant because the path coefficient between two variables was at $1.705(t=5.279, P<0.001)$.

Hypothesis 3-1 'Community spirit in school sports club participants will have an impact on positive sharing, subfactor of empathy' appeared statistically significant because the path coefficient between two variables was at $0.291(t=2.089, P<0.05)$ and so adopted. 'Community spirit in school sports club participants will have an impact on emotional contagion, subfactor of empathy' appeared not statistically significant because the path coefficient between two variables was at $-0.027(t=-0.110, P=0.912)$ and so dismissed. Also, 'Community spirit in school sports club participants will have an impact on emotional involvement, subfactor of 
empathy' appeared not statistically significant because the path coefficient between two variables was at $0.057(t=0.370, P=$ $0.711)$.

\section{Involvement in the relationship between the level of humanity and empathy}

This study examined the relation between personality and empathy depending on involvement in school sports club participation. To verify the goodness-of-fit of the model showing the relation between personality and empathy, this study conducted a structural model analysis. In the model of low involvement group, $\chi^{2}=310.633, d f=195, P=0.000, \chi^{2} / d f=1.593, \mathrm{TLI}=0.903$, $\mathrm{CFI}=0.918$, and RMSEA $=0.066$; in the model of intermediate involvement group, $\chi^{2}=259.718, d f=175, P=0.000, \chi^{2} / d f=$ $1.484, \mathrm{TLI}=0.914, \mathrm{CFI}=0.928$, and $\mathrm{RMSEA}=0.063$; and in the model of high involvement group, $\chi^{2}=172.542, d f=120, P=$ $0.001, \chi^{2} / d f=1.438, \mathrm{TLI}=0.919, \mathrm{CFI}=0.936$, and $\mathrm{RMSEA}=$ 0.055 , which suggested that the goodness of fit was relatively satisfied.

The result of identifying the causal relation between personality and empathy depending on the level of low involvement among school sports club participants is shown in Table 3. In low involvement group, confidence and community spirit, subfactors of personality appeared not having an impact on subfactors of empathy $(P>0.05)$, but positive attitude of personality appeared having a positive impact on all three factors such as positive sharing indicating $1.291(t=2.253, P<0.05)$, emotional contagions indicating $3.619(t=2.711, P<0.01)$, and emotional involvement indicating $2.670(t=2.751, P<0.05)$.

The causal relation between personality and empathy depending on the level of intermediate involvement among school sports club participants is shown in Table 4. In intermediate involvement group, confidence and community spirit, subfactors of personality appeared not having an impact on subfactors of empathy $(P>0.05)$. However, positive attitude, subfactor of personality appeared having a positive impact on all three factors of empathy such as positive sharing indicating $3.88(t=3.438, P<0.001)$, emotional contagion indicating $1.341(t=4.707, P<0.001)$, and emotional involvement indicating $0.794(t=4.553, P<0.001)$. Both the low involvement group and intermediate involvement group showed

Table 3. Causal relation between personality and empathy depending on the level of low involvement

\begin{tabular}{|c|c|c|c|c|c|c|}
\hline Hypothesis & & & Path coefficient & Standard error & $t$-value & Adopted or dismissed \\
\hline Self-confidence & $\rightarrow$ & Emotional positive sharing & 0.086 & 0.262 & 0.329 & Dismissed \\
\hline Self-confidence & $\rightarrow$ & Emotional contagion & -0.827 & 0.594 & -1.392 & Dismissed \\
\hline Self-confidence & $\rightarrow$ & Emotional involvement & -0.422 & 0.429 & -0.985 & Dismissed \\
\hline Positive attitude & $\rightarrow$ & Emotional positive sharing & 1.291 & 0.573 & $2.253^{*}$ & Adopted \\
\hline Positive attitude & $\rightarrow$ & Emotional contagion & 3.619 & 1.335 & $2.711^{* *}$ & Adopted \\
\hline Positive attitude & $\rightarrow$ & Emotional involvement & 2.670 & 0.971 & $2.751^{* *}$ & Adopted \\
\hline Community spirit & $\rightarrow$ & Emotional positive sharing & -0.231 & 0.246 & -0.938 & Dismissed \\
\hline Community spirit & $\rightarrow$ & Emotional contagion & -0.537 & 0.507 & -1.059 & Dismissed \\
\hline Community spirit & $\rightarrow$ & Emotional involvement & -0.359 & 0.371 & -0.968 & Dismissed \\
\hline
\end{tabular}

${ }^{*} P<0.05 .{ }^{* *} P<0.01$.

Table 4. Causal relation between personality and empathy depending on the level of intermediate involvement

\begin{tabular}{|c|c|c|c|c|c|c|}
\hline Hypothesis & & & Path coefficient & Standard error & $t$-value & Adopted or dismissed \\
\hline Self-confidence & $\rightarrow$ & Emotional positive sharing & 0.175 & 0.168 & 1.038 & Dismissed \\
\hline Self-confidence & $\rightarrow$ & Emotional contagion & -0.118 & 0.326 & -0.362 & Dismissed \\
\hline Self-confidence & $\rightarrow$ & Emotional involvement & 0.159 & 0.204 & 0.779 & Dismissed \\
\hline Positive attitude & $\rightarrow$ & Emotional positive sharing & 0.388 & 0.113 & $3.438^{* * *}$ & Adopted \\
\hline Positive attitude & $\rightarrow$ & Emotional contagion & 1.341 & 0.285 & $4.707^{* * *}$ & Adopted \\
\hline Positive attitude & $\rightarrow$ & Emotional involvement & 0.794 & 0.174 & $4.553^{* * *}$ & Adopted \\
\hline Community spirit & $\rightarrow$ & Emotional positive sharing & 0.350 & 0.201 & 1.742 & Dismissed \\
\hline Community spirit & $\rightarrow$ & Emotional contagion & -0.384 & 0.371 & -1.034 & Dismissed \\
\hline Community spirit & $\rightarrow$ & Emotional involvement & -0.126 & 0.227 & -0.553 & Dismissed \\
\hline
\end{tabular}

${ }^{* * *} P<0.001$. 
Table 5. Causal relation between personality and empathy depending on the level of high involvement

\begin{tabular}{lllcccc}
\hline Hypothesis & & & Path coefficient & Standard error & $t$-value & Adopted or dismissed \\
\hline Self-confidence & $\rightarrow$ & Emotional positive sharing & -1.772 & 0.792 & $-2.237^{*}$ & Withheld \\
Self-confidence & $\rightarrow$ & Emotional contagion & -3.033 & 1.525 & $-1.990^{*}$ & Withheld \\
Self-confidence & $\rightarrow$ & Emotional involvement & -2.809 & 1.541 & -1.823 & Dismissed \\
Positive attitude & $\rightarrow$ & Emotional positive sharing & 1.162 & 0.632 & 1.838 & Dismissed \\
Positive attitude & $\rightarrow$ & Emotional contagion & 3.347 & 1.341 & $2.497^{*}$ & Adopted \\
Positive attitude & $\rightarrow$ & Emotional involvement & 2.693 & 1.325 & $2.033^{*}$ & Adopted \\
Community spirit & $\rightarrow$ & Emotional positive sharing & 0.744 & 0.366 & $2.031^{*}$ & Adopted \\
Community spirit & $\rightarrow$ & Emotional contagion & 0.469 & 0.622 & 0.754 & Dismissed \\
Community spirit & $\rightarrow$ & Emotional involvement & 0.601 & 0.545 & 1.102 & Dismissed
\end{tabular}

${ }^{*} P<0.05$.

the same result patterns.

The causal relation between personality and empathy depending on the level of high involvement among school sports club participants is shown in Table 5. In high involvement group, confidence, subfactor of personality appeared having a negative impact on two factors of empathy such as positive sharing indicating $-0.772(t=2.237, P<0.05)$ and emotional contagion indicating $-3.033(t=-1.990, P<0.05)$. Also, positive attitude had a positive impact on two factors of empathy such as emotional contagion indicating $3.347(t=2.497, P<0.05)$ and emotional involvement indicating $2.693(t=2.033, P<0.05)$ and community spirit appeared having a positive impact on positive sharing indicating 0.744 ( $t=2.031, P<0.05)$. Resultingly, the high involvement group showed different result patterns from the low and intermediate involvement groups.

\section{DISCUSSION}

This study analyzed the relation between personality and empathy depending on Korean teenagers' school sports club involvement and obtained the following results. Positive attitude, one of personality factors appeared to have an impact on all three subfactors of empathy and confidence, one of personality factors appeared to have a negative (-) impact on emotional contagion and emotional involvement, subfactors of empathy among the teenagers who participated in sports club. In addition, community spirit appeared to have a positive impact on emotional contagion, subfactor of empathy. This suggests that there is a relation between personality and empathy among adolescents.

To look at it more closely, the fist thing is that there is a relations between personality and empathy. This study verified the relation between personality and empathy depending on the level of school sports club involvement. As a result, there was a differ- ence in confidence and community spirit, subfactors of personality in high involvement group in school sports club compared to the low and intermediate involvement groups. In all groups, positive attitude appeared to be related to empathy. In other words, what can be predicted from this study is that the empathy skills can be improved by participating in personality programs that can increase positive attitude regardless of the intensity of involvement in school sports club among students.

Empathy plays an important role in humans' social behaviors. Empathy prevents us from doing harm to others and induces altruistic behavior. Also, it plays a deciding role in cultivating emotional bond between mother, sibling, spouse, and bigger social group members (Singer, 2006). Such an empathy can have an impact on positive mind and living attitude. In other words, as positive attitude, subfactor of personality means having a positive mind and attitude in all situations, it may have an impact on empathy skills such as emotional contagion, emotional involvement, and positive sharing. In particular, community spirit, subfactor of personality had a positive impact on emotional contagion factor of empathy. As community spirit means consciousness or emotion among members in communal society and is in the same context as the emotional contagion of the empathy that may have equal feelings as other people have, mutual relation seems to be present. In addition, confidence factor of personality is a representative factor that's acquired by sports participation (Cha et al., 2013). So far, it's been reported that confidence is a representative emotional change factor by teenagers' sports participation and that sports participation improves confidence with interpersonal relation (Kim, 2010; Motl et al., 2001). Also, teenagers' confidence is a very important factor as it acts more positively and productively in family, school, and local community. However, confidence factor appeared to have a negative impact on emotional contagion and emotional involvement, subfactors of empathy. It is attribut- 
able to the fact that confidence is the degree of mindset that one is able to do anything and implies that one is willing to take the lead and thus emotional contagion and emotional involvement, subfactors of empathy has the aim of understanding others rather than self as both mean forming the same emotion as others. Shin and $\operatorname{Kim}$ (2003) also supported this study by reporting that the adolescents who actively participated in physical activities showed lack of cooperation with others due to excessive confidence with others in the process of forming a relationship with others and rather had a negative impact on interpersonal relations. In other words, it's interpreted that too excessive confidence of an individual generated by sports participation may rather be an inhibiting factor in forming a sympathy with others.

Smith (2003) reported that school PE activity participation cultivates positive personality and morality through interaction among students, enables people to form a desirable sociality, forms a wider peer relation in terms of interpersonal relation. In other words, it means that adolescents have a positive impact on interpersonal relation as positive personality and morality are developed through the physical activities that students participate in groups in school. Such an empathy contributes to integration, harmony, and communication of interpersonal relation as it comes from interest in other people. In group culture putting stress on 'relation' like in Korea, empathy becomes an important factor in interpersonal relation. To look at a study targeting Korean college students (Heo and Lee, 2010), empathy appeared to have improved self-respective and subjective well-being. This suggests that empathy plays an important role in an individual's wellbeing as well as treatment and interpersonal relation in Korean culture. Therefore, it's considered that empathy with others is basic to and core factor for personality in communal society. Such an empathy is formed relatively in childhood (Chakrabarti et al., 2006) and empathy is associated with physical aggressiveness (Yeo et al., 2011). So to reduce such aggressiveness as school violence, it's necessary to have an empathy training relatively in childhood.

So far, sports activity has been evaluated as an effective activity for solving the problems that are caused by academic background-oriented entrance exam and consumption- and pleasure-seeking-oriented leisure culture and implementing the images of physically, psychologically, and socially healthy adolescents. Deep breathing and concentration through exercise induces to activate parasympathetic nerve, relaxes muscle and nervous system, causes stable state of mind and body, and reduces aggressiveness, which ultimately may have a positive impact on personality and empathy (Krämer et al., 2010; Shamay-Tsoory et al., 2009).
In addition, Donaldson and Ronan (2006) reported that as teenagers' sports participation improved, it had a positive impact on reduced problematic behavior as well as emotional purification.

The second thing is involvement in the relationship between the level of humanity and empathy. In particular, this study verified the relation between personality and empathy depending on the level of school sports club involvement. As a result, in all involvement groups who participated in school sports club, positive attitude, personality factor appeared to be associated with empathy. Therefore, what can be predicted from this study is that if the students who participated in school sports club participate in the personality programs that may increase positive attitude regardless of the intensity of involvement, their empathy skills can be improved.

In addition, high involvement group in school sports club showed different patterns in confidence and community spirit, personality factors, from low involvement group and intermediate involvement group. Like this, when it comes to the difference in the degree of sports participation, students' empathy skills become higher when the frequency of participants' participation in physical activities, in other words, frequency of participation is higher. Therefore, it's in the same context as this study that high involvement group who participated in school sports club showed a difference from low and intermediate involvement groups. To look at such results specifically, confidence appeared to have a negative impact on positive emotion and emotional contagion in high involvement group. This is an interesting result in that confidence, personality factor of the students who had a high involvement level served as negative factor for empathy factors. In other words, confidence, which was generated by school sports club participation showed a negative association with emotional contagion and emotional involvement. Cha and Kim (2014) who investigated the difference in personality among teenagers depending on the characteristics of school sports club participation also reported that sports participation-induced personality showed a higher personality (confidence) when school sports club participation frequency is higher and degree of participation is more active. Therefore, it's considered that when the level of sports participation is high, subsequently personality (confidence) is cultivated. Park (2013) demanded that for personality education, too excessive competition should be avoided in school PE, which also supports this study. Such results are suggestive of the future direction of school sports club. In other words, the educational direction that school sports club should follow is that strategic approaches are required, for example, selecting non-competitive events that may 
have an impact on the cultivation of empathy skills, beyond just fostering an individual's personality. In other words, it's considered that it seems to be necessary to apply the events into the direction of incorporating school sports club program into the educational course which is based on cooperation and collaboration. Also, personality education program through school sports club participation should be applied to school field when it's designed based on such results.

Through this study, it can be concluded that educational program should be organized with that physical activities that may improve personality and empathy rather than the sports activities having excessive competitive elements for students who participate in school sports club. In other words, school sports club will have to be an activity that students can be influenced in a positive way, physically or psychologically and understand and take good care of each other. Based on such results, various sports participation policies for adolescents that can be used to maintain and improve interpersonal relations by understanding and taking care of other people as well as maintaining and enhancing health in the participation process are required; appropriate programs should be developed; and strategic supply of such programs depending on the level of personality and empathy should be implemented.

Adolescence is a period that adolescents are physically and mentally developed in terms of personality, morality, creativity, and sociality. During this period, learning and experience are very important for lifetime. Therefore, teenagers' school sports club activity can contribute to fostering positive and desirable personality and stabilizing emotional empathy among student participants. This study identified the relation between personality and empathy in adolescents, which was not studied before. As presented in previous researches, it can be found that positive personality can be cultivated by teenagers' communal activities in school life. The link to connect this with empathy factor is considered to be the very communal activity, sports. Therefore, this study aimed to identify the causal relation between personality and empathy through SEM analysis as follows:

First, as a result of verifying the causal relationship between personality and empathy, confidence, subfactor of personality had a negative (-) impact on emotional contagion and emotional involvement, subfactors of empathy. It appeared that positive attitude, subfactor of personality had a significant impact on positive sharing, emotional contagion, and emotional involvement, subfactors of empathy and that community spirit, subfactor of personality had a significant impact on positive sharing, subfactor of empathy.
Second, the variables that showed a statistical difference depending on the level of school sports club participation included confidence, positive attitude, and community spirit as subfactors of personality and positive sharing, emotional contagion, and emotional involvement as subfactors of empathy.

Empathy can be seen as an important factor for improving communication skills in the modern society emphasizing communication skills (Singer, 2006) and in particular, arousing empathy is very important in adolescent period when adolescents are affected a lot by others and imitate others. Therefore, this study highlighted the necessity and importance of educational programs that can be used to conduct righteous personality education and form emotional empathy for students. Such school sports club programs are not for simply fostering individual personality but require systematic programs in the direction of improving positive attitude. So subsequent appropriate school sports club participation guidelines need to be presented.

This study has some suggestions for further studies. First, as this study conducted a cross-sectional study in students who participated in after-school sports club activities, limited understanding and interpretation are required, and it will be valuable if further studies conduct a longitudinal analysis of the continuously changing process of research subjects. Second, this study did not consider the difference in characteristics of individual exercise ability in student participants and the difference in the events that they participated. So further studies need to classify the difference in the characteristics of individual exercise ability and the events that they participated among the students who participated in after-school sports club in more detail.

\section{CONFLICT OF INTEREST}

No potential conflict of interest relevant to this article was reported.

\section{REFERENCES}

Anderson D. Recovering humanity: movement, sport, and nature. J Philos Sport 2001;28:140-150.

Anderson JC, Gerbing DW. Structural equation modeling in practice: a review and recommended two-step approach. Psychol Bull 1988;103: 411-423.

Barr JJ, Higgins-D'Alessandro A. Adolescent empathy and prosocial behavior in the multidimensional context of school culture. J Genet Psychol 2007;168:231-250. 
Bennett G, Ferreira M, Lee J, Polite F. The role of involvement in sports and sport spectatorship in sponsor's brand use: the case of mountain dew and action sports sponsorship. Sport Mark Q 2009;18:14-24.

Bierhoff HW, Rohmann E. Altruistic personality in the context of the empathy-altruism hypothesis. Eur J Pers 2004;18:351-365.

Caspi A, Roberts BW. Personality development across the life course: the argument for change and continuity. Psychol Inq 2001;12:49-66.

Cha EJ, Kim YJ. Analysis of difference in sports personality in adolescents who participated in school sports clubs. J Korea Sports Assoc 2014;53: 25-34.

Cha EJ, Kim YJ, Heo JH. Personality evaluation tool validation test for teenagers who participated in sports. J Korea Soc Sports Sci 2013;22: 361-373.

Chakrabarti B, Bullmore E, Baron-Cohen S. Empathizing with basic emotions: common and discrete neural substrates. Soc Neurosci 2006;1: 364-384.

Decety J, Jackson PL. The functional architecture of human empathy. Behav Cogn Neurosci Rev 2004;3:71-100.

Donaldson SJ, Ronan KR. The effects of sports participation on young adolescents' emotional well-being. Adolescence 2006;41:369-389.

Donnerstein E, Donnerstein M, Munger G. Helping behavior as a function of pictorially induced moods. J Soc Psychol 1975;97(Second Half):221225 .

Eisenberg N, Miller PA. The relation of empathy to prosocial and related behaviors. Psychol Bull 1987;101:91-119.

Feshbach ND, Feshbach S. Empathy training and regulation of aggression: potentialities and limitations. Acad Psychol Bull 1982;4:339-413.

Findlay LC, Girardi A, Coplan RJ. Links between empathy, social behavior, and understanding in early childbood. Early Child Res Q 2006;21: 347-359.

Gano-Overway LA, Newton M, Magyar TM, Fry MD, Kim MS, Guivernau MR. Influence of caring youth sport contexts on efficacy-related beliefs and social behaviors. Dev Psychol 2009;45:329-340.

Gini G, Albiero P, Benelli B, Altoè G. Does empathy predict adolescents' bullying and defending behavior? Aggress Behav 2007;33:467-476.

Gordon M. Roots of empathy: changing the world child by child. New York: Workman Publishing; 2009.

Heo J, Lee C. An impact of empathy on self-respect and subjective wellbeing. J Acoust Soc Korea 2010;29:332-338.

Kim SW. An impact of college students' sports club participation on interpersonal relation satisfaction and college life satisfaction as social physical activity. J Sport Leis Stud 2010;39:887-897.

Krämer UM, Mohammadi B, Doñamayor N, Samii A, Münte TF. Emotional and cognitive aspects of empathy and their relation to social cognition-an fMRI-study. Brain Res 2010;1311:110-120.
Leming JS. Tell me a story: an evaluation of a literature-based character education programme. J Moral Educ 2000;29:413-427.

Lickona $\mathrm{T}$. Teaching respect and responsibility reclaiming children and youth. J Emot Behav Probl 1996;5:143-151.

Mehrabian A, Epstein N. A measure of emotional empathy. J Pers 1972; 40:525-543.

Ministry of Culture, Sports and Tourism. Major mission plans for school physical education in 2013-happiness eucation that fosters dream and talent. Sejong (Korea): Ministry of Culture, Sports and Tourism; 2013.

Ministry of Education. Education contents and mothods and evaluative system innovation plans for fostering cteativity and personality. Sejong (Korea): Ministry of Education; 2010.

Ministry of Education. School physical education activation plans. Sejong (Korea): Ministry of Education; 2008.

Ministry of Education. School physical education major tasks planning. Sejong (Korea): Ministry of Education; 2013.

Motl RW, Dishman RK, Saunders R, Dowda M, Felton G, Pate RR. Measuring enjoyment of physical activity in adolescent girls. Am J Prev Med 2001;21:110-117.

Newcombe PA, Boyle GJ. High school students' sports personalities: variations across participation level, gender, type of sport, and success. Int J Sport Psychol 1995;26:277-294.

Park JJ. Reflective considerations of sports competition mechanism for fostering personality. Curric Stud 2013;31:281-297.

Park SM, Heo SH. Development of integrative personality scale for adolescents. Child Educ 2012;21:35-47.

Preston SD, de Waal FB. Empathy: its ultimate and proximate bases. Behav Brain Sci 2002;25:1-20.

Roberts BW, Caspi A, Moffitt TE. Work experiences and personality development in young adulthood. J Pers Soc Psychol 2003;84:582-593.

Shamay-Tsoory S, Harari H, Szepsenwol O, Levkovitz Y. Neuropsychological evidence of impaired cognitive empathy in euthymic bipolar disorder. J Neuropsychiatry Clin Neurosci 2009;21:59-67.

Shin CB, Kim JM. A study on the effect of interpersonal relation program through physical activity. Youth Stud Res 2003;10:21-47.

Singer T. The neuronal basis and ontogeny of empathy and mind reading: review of literature and implications for future research. Neurosci Biobehav Rev 2006;30:855-863.

Smith AL. Peer relationships in physical activity contexts: a road less traveled in youth sport and exercise psychology research. Psychol Sport Exerc 2003;4:25-39.

Wankel LM, Kreisel PS. Factors underlying enjoyment of youth sports: sport and age group comparisons. J Sport Psychol 1985;7:51-64.

Wiley CG, Shaw SM, Havitz ME. Men's and women's involvement in sports: an examination of the gendered aspects of leisure involvement. 
Leis Sci 2000;22:19-31.

Yeo LS, Ang RP, Loh S, Fu KJ, Karre JK. The role of affective and cognitive empathy in physical, verbal, and indirect aggression of a Singaporean sample of boys. J Psychol 2011;145:313-330. 